\title{
Der Gehalt des Lichtes an Ultraviolett.
}

\author{
Eine Entgegnung an Herrn Sanitätsrat Dr. Fritz Schanz.
}

\author{
Von \\ Professor: Dr. phil. C. Domo, \\ Davos.
}

Zum fünften Male muß ich zur Feder greifen, um Angriffe des Herrn Sc hanz gegen meine Meßmethoden abzuwehren. In den von Herm S. auf $\mathrm{S}$. 162 des Bd. 103, Heft 2, dieser Zeitschrift in Anmerkung zitierten Zeitschriften findet der Leser meine stets prompt erfolgten Widerlegungen, desgleichen in Arch. $f$. d. ges. Physiol. 184. Herr S. benutzt die Gelegenheit der Veröffentlichung einer mit einer kostbaren, aber schwierig zu behandelnden Apparatur angestellten Untersuchung über ultraviolettes Licht, um in mehr persönlicher als sachlicher Weise seine längst abgeschlagenen Angriffe gegen meine Meßmethoden $\mathrm{zu}$ wiederholen. Ich verweise auf die oben zitierten Entgegnungen und füge hinzu:

Ganz unmöglich ist es ein Beweis für die Fehlerhaftigkeit physikalischer Methoden, wenn ihre Resultate mit den biologischen Beobachtungen nicht in Einklang stehen (der Haupteinwurf, den Herx S. immer wieder gegen meine Meßmethoden heranzieht), Anfgabe der Biologie ist es vielmehr, den in der belebten Materie liegenden Ursachen nachzugehen, aus welchen nicht die auf Grund der definierten, physikalischen Größen gemutmaßten, sondern andere Reaktionen folgen ${ }^{\mathbb{I}}$ ). Eine andere Ansicht hierüber würde doch kaum etwas anderes bedeuten, als Ursache und Wirkung verweehseln. Bis zu welchen Spekulationen sich Herr S. hinreiBen läßt, um in ganz unberechtigtem RückschluB , aus biologischen Beobachtungen" physikalische Größen, die ultraviolette Strahlung in den Tropen und an den Polen, abzuleiten, das möge, wer sich ein rechtes Bild über die Art seiner Beweisfühmung bilden will, nachlesen in der Wiener klin. Rundschan XXXIV, Jahrgang 1920, Heft 3/6. Aus den gegenwärtig abgeschlossenen Untersuchungen hält Herr S. für bewiesen (S. 174), "die ultravioletten Strahlen sind es, welche das Leben auf dem Erdball erzengt haben and vor allem es unterhalten", andererseits wiederum sind sie nach seiner Meinung in Beziehung auf das Auge, des Menschen kostbarstes Organ, nur "Verunreinigungen des Liehtes ${ }^{\text {"s }}$ (S. 175).

Herr Dr. Bernhard hat sich keineswegs in dem Sinne geäuBert, daß meine Resultate nicht zutreffen, sondern sich mit Gegenüberstellung der verschiedenen

1) Anerkennenswert ist W. Filehnes Verhalten, welcher sein im Eifer abgegebenes Urteil „Rayleighs Gesetz, so richtig es mathematisch abgeleitet ist, entspricht nicht den Tatsachen" in der Physikal. Zeitschr. 1920 S. 351, widerruft und seine dem Gesetz anscheinend widersprechenden Befunde durch dic spezifische physiologischo Wirkung auf das menschliche Sehorgan erklärt. 
Befunde begnügt derart, daß nach seinen Beobachtungen an Patienten die ultraviolette Strahlungsintensität im Frühjahr stärker als im Herbst sei, während sie hier in länger als 10 jährigen Messungen dauernd im Herbst stärker als im Frühjahr gefunden ist. Der Grund der Diskrepanz ist, wie ich sehon in meiner Schrift, „Klimatologie im Dienste der Medizin", Viewegs Tagesfragen, Heft 50, S. 48, erklärt habe, die Lichtentwöhnung des Menschen von besonders stark Pigment erzeugenden ultravioletten Strahlenarten während des niedrigen Standes der Sonne. Herr $\mathrm{S}$. ist auf die den Beweis hierfür erbringenden Tabellen 19-23 meiner "Studie über Licht und Luft des Hochgebirges", Vieweg 1911, welche die bedeutenden Schwankungen des ultravioletten Spektrumendes in Abhängigkeit von Tages- und Jahreszeit zahlenmäBig auf Grund von mehrjährigen täglichen Dauerregistrierungen nachweisen, sehon wiederholt hingewiesen, aber ohne jeden Erfolg. Sie deuten mehr als alle bisher erbrachten Untersuchungsresultate darauf hin, wo die biologisch wirksamsten Strahlen zu suchen sind, und ein verblüffend klarer, auf Laboratoriumsversuchen beruhender Beweis für die Richtigkeit dieses Hinweises wird demnächst aus berufener physikalischer Feder veröffentlicht werden und die ganze Frage der Pigmentbildung, soweit bei ihr die Strahlung auslösend wirkt, in bester Ưbereinstimmung mit meinen soeben zitierten Tabellen klären und gleichzeitig einen glänzenden Beweis dafür bringen, wie notwendig, da wegweisend für die Medizin, solche freilich, wenn exakt ausgeführt, sehr mühsamen und viel voraussetzenden physikalisch-meteorologischen Untersuchungen sind. Eine dieser wichtigen Tabellen ist übrigens in dem genannten leicht zugänglichen Heft 50 der Sammlung Vieweg auf S. 44 abgedruckt.

Herr S. sucht die biologisch wirksamsten Strahlen in dem ganzen Spektralbezirk zwischen 400 und $300 \mu \mu$ (S. 173), kleinere Wellenlängen als $300 \mu \mu$ wirken seiner Meinung nach nur destruktuierend auf die lebende Substanz (ob der hierfür von ihm erbrachte Beweis genügt, mag jeder selbst entscheiden). Die $\mathrm{Ab}$ grenzung bei $400 \mu \mu$,dürfte in physiologischer Hinsicht den Verhältnissen gut entsprechen “ - Begründung (S. 162). „Hört auch erst bei $392 \mu \mu$ die Sichtbarkeit des Lichtes auf, so beginnt es schon im blauen und violetten Spektralbereich unsichtbar zu werden“ (sic!) und ,Unsere Linse ist von Jugend auf gelblich gefärbt und verrät dadurch, daß schon Strahlen aus dem blauen und violetten Wellenlängenbereich unsichtbar sind". Da müßte Herr $\mathrm{S}$. die Grenze doch schon ins Blauviolett verlegen und dann könnte es für seine Meßzwecke ja gar kein besseres Instrument als das von ihm bemängelte Zinkkugelphotometer geben, denn es spricht noch zu einem kleinen Teil (etwa 10\%) auf blauviolette, im übrigen auf die gesamte ultraviolette Strahlung an, welche nach Herrn S's. jetzt angestellten (zu anderen Beobachtungen nicht gut stimmenden) Untersuchungsresultaten in der Ebene bei $300 \mu \mu$ praktisch aufhört, in der Höhe aber - wie aus den oben erwähnten Tabellen 19-23 hervorgeht - im Mittel weiter reicht. Im übrigen ist das in den Jahren 1908-1911 hier benutzte Zinkkugelphotometer lange überholt durch die viel exaktere, auch von Herm S. jetzt angewandte photoelektrische Zelle, und die von mir mit dieser Methode seit 1914 in sechs verschiedenen Spektralteilen (mit den optischen Schwerpunkten 465, 415, 405, 400, 379, $315 \mu \mu)$ erhaltenen Resultate können Herrn S. nicht unbekannt gewesen sein, da er mein großes Werk ,Himmelshelligkeit, Himmelspolarisation und Sonnenintensität Davos 1911-1918“, Abhandlungen des PreuB. Meteorolog. Inst. 6, zitiert.

Herr S. behauptet $S .167$, ich hätte in meiner Abweisung seiner Abbandlung „Höhensonne" in der „Strahlentherapie "4 (8, S. 609) bezweifelt, daß es möglich wäre, so geringe elektrische Kräfte, wie sie bei Spektrallinienbreite photoelektrisoh ansgelöst werden, exakt $z u$ messen. In Wirklichkeit habe ich geschrieben; 
,Auf galvanometrischem Wege, welcher für über Jahre hinweg zu führende MeBreihen und für Registrierzwecke der allein gegebene ist, kann die Kaliumzelle nicht Aufschluß geben." Liegt bei dieser wichtigen Omission wirklich anch nur ein Lapsus von Herrn S. vor? DaB man mit der von Herm S. angewandten Auflademethode etwa 1000 mal kleinere, bei Beobachtung der Fadensprünge sogar 100 Millionen mal kleinere Strahlungsintensitäten messen kann, ist jedem Eingeweihten bekannt und in dem für weitere Kreise von mir geschriebenen Büchlein über „Physik der Sonnen- und Himmelsstrahlung", Sammlung Vieweg, 63, leicht nachzulesen; ebenso belkannt ist aber auch, dab diese Methoden nicht za Registriermethoden ausgebildet werden können, welche absolut nötig sind, wenn man die Apparatur in den Dienst der meteorologischen Optik stellen will und sioh nicht mit einer einzelnen oder vielmelr zwei Messungen begnügt, wie Herr $\mathbf{S}$. sie für die vorliegende Arbeit angestellt hat.

Sohließlich behauptet Herr S. (S. 174), ,meine Werte für die blauviolette Strahlung sind viel zu hoeh, die Werte für die ultraviolette viel zu niedrig:" Diese Behauptung von Herm $\mathbf{B}$. ist neu und eigenartig, denn nirgend in meinen seit 10 Jahren herausgegebenen zahlreichen Veröffentlichungen habe ich eine Relation zwischen meinen blauvioletten und ultravioletten Strahlungsgrößen angefürt und anführen können, da jedes Verbindungsmaß fehlt. Herr S. seheint wiederum, ohne irgendeinen Beweis dafür bringen zu können, behaupten zu wollen, die in Davos für die ultraviolette Sonnenstrahlung mit 10:1 gefundene Amplitude zwischen Sommer- und Wintermittagswerten sei zu gro, , - die inzwischen im Flachlande (in Kolberg) gemessene ist - wie zu erwarten war - noch viel größer, nämlich $46,7: 1$, auch haben die Kolberger Messungen bestätigt, daß im Ultraviolett die Herbstwerte weit größer sind als die Frühjahrswerte.

Herm S. ist eine solche Beziebung auf die Energieeinheit mittels des Thermoelementes bei seiner kostbaren Apparatur möglich. Abweichend von sonstigem Gebrauch bei Veröffentlichungen von mit so komplizierter Apparatur angestellten Arbeiten gibt er leider keine Einzelheiten an über die Dispersion des Monochromators, Grenzen der Aufladegeschwindigkeit und des Aufladebereichs, Kapazität, Empfindlichkeit des Elektrometers und der zu ihrer Regulierung verwandten Hilfsmittel, Nullage, Troekenvorrichtung zur Verhinderung galvanischer Ströme an der Kontaktstellen, Sohutzmittel gegen die Erhitzung der Zelle bei Exposition des Instrumentes gegen die Sonne oder die teilweise sehr starken Liehtquellen (3000 kerzige Nitralampe), ja, man vermiBt sogar Angaben über die angewandte Zelle (ob hochevaluiert oder gasgefüllt, mit welcher Gasfüllung, ob mit kugelförmiger oder ebener Kathode). Da er nach seinen Angaben in allem vom Phy. sikalisehen Institut der Dresdner Hochschule beraten war, können wir ja freilich annehmen, daß es ihm gelungen ist, sich in die für einen Nichtphysiker recht sohwer zu meisternde Arbeitsmethode genügend sicher zu finden. Unerwartet ist dann aber, wem Herr $\mathrm{S}$. sioh wirklich ganz in die Naterie hineingelebt hat, daß er nun nicht die wertvollsten Vorteile aus seiner Apparatur zieht und seine Resultate niebt in absoluten EnergiemaB meldet und diskutiert, sondem in einem willkïrlichen, allein von der Empfindlichkeit der ron ihm gewählten Zelle abhängigen. Alle seine Kurven auf S. 170 wären, wenn er zu ihrer Korrektur die der Kurve der Abb. 5 auf S. 169 zu Grunde liegenden Zahlen zu benutzen gewuBt hätte, ihrer Gestalt nach leicht und sicher vergleichbar gewesen mit den an anderen Orten gefundenen, und er hätte sogar alle im absoluten Maßs anführen Ioönnen, d. $h$. auch dem Absolutwert nach vergleichbar mit Befunden an anderen Orten, an welohen nach gleicher Methode gearbeitet wird. Freilich wäre dann, wie auch trotz des Kleinen MeBstabes dex. Kurven mit Sicherheit dureh Umrechnung nachzuweisen jst, sofort aufgefallen, daB sich Hers \&. mit seiner Tntensitatskurve 
der Sonnenstrahlung in unaufklärbaren Widerspruch setzt nit allen bisherigen Befunden, mögen sie auf photometrischem, photoelektrischem, photographisebem oder kalorimetrischem Wege erhalten sein, insbesondere auch mit den seit 1909 am Weltmusterinstitut, dem Astrophysical Observatory of the Smithsonian Institution der Vereinigten Staaten, regelmäBig durchgeführten Messungen, nach welchen ein Anstieg der Intensität zwischen 450 und $370 \mu \mu$ (wie er aus der Kombination der Abb. 5 und der Kurve $I$ in Abb, 6 hervorgeht) unmöglich ist. Auch die Intensitätskurve des Quarzlichtes scheint mir mit der von E. Ladenburg ermittelten sehr schwer vereinbar zu sein, wenn es hier auch nicht möglich ist, einen absoluten Beweis gegen die Riehtigkeit von S.s Kurve zu erbringen.

Vorausgesetzt aber, seine Resultate wären richtig, wie ist es nur Herrn S. möglich, aus seinèn zueinander doch in festem relativen $\mathrm{MaB}$ stehenden Kurven herauszulesen und gesperrt zu drucken und auf Grund dieses gesperrt gedruckten Satzes eine heftige Polemik gegen einen Kollegen der Ophthalmologie einzuleiten. „Die Strahlen von 400 bis $300 \mu \mu$ sind im Sonnenlicht viel intensiver vertreten als im Lichte jeder künstlichen Lichtquelle" (S. 173). Zwischen etwa 320 und $300 \mu \mu$ ist nach Abb. 6 das von ihm angewandte Quarzlicht 10 bis 20 mal intensiver als das Sonnenlicht um die Mittagszeit des 17. Juni. Femer: Womit ist, ,bewiesen“, daß die Quarzlampe gerade mit ihren Linien bei etwa $220 \mu \mu$ destruktuierend wirkt? (S. 173). Man kann es wohl mutmaßen, aber eine Mutmaßung ist doch kein Beweis, welcher im übrigen bei genügendem Geldaufwand für geeignete Apparatur (mit genügend großen und weit dispergierenden Quarzprismen) durch das Experiment an der Haut des lebenden Mensehen leicht positiv oder negativ zu erbringen wäre.

Bei solchen Schlußfolgerungen kann sich Herr S. nicht wundern, wenn seine Fachkollegen, wie er klagt, seine Lichtlehre nicht anerkennen. Der gleiche Mangel an Unterscheidung zwischen Behauptung und Beweis ist es ja auch, was mich hier zur persönliehen Abwehr und gleichzeitig zur Kritik von Herm S.s vielfach zu korrigierenden Resultaten zwingt - eine wenig fruchtbare, aber notwendige Aufgabe. Als Trost für solche aufgezwungene Arbeit dient die ziemlich sicher gewordene Aussicht, von der ich hier Mitteilung zu machen Gelegenheit nehme, daß es den hjesigen 5 jährigen, von den Begründern der photelektrischen Methode, Herren Elster und Geitel, emsig unterstützten Bemühungen gelingen wird, eine unter allen Bedingungen zuverlässige Anordnung zur dauernden Registrierung der ultravioletten Intensität der Sonnen- und auch der Himmelsstrahlung $\mathrm{zu}$ treffen, viel schwieriger durchführbar als die analogen der Dauerregistrierung der Helligkeit, von welcher in diesen Tagen die Meteorologische Zeitschrift das erste Jahresresnltat wiedergibt, der ersten zuverlässigen Helligkeitsregistriermethode, wie nicht $z$ viel behauptet ist, welche daher auch prompt von den Herren Amerikanern übernommen wurde, nachdem ich die erste Beschreibung der Methode in der Monthly Weather Review Juni 1920 gebracht hatte.

Zum SchluB wird mir in Anbetracht der vier Jahre hindurch von Herrn S. gegen meine Arbeitsdomäne gerichteten, gänzlich umbegründeten Angriffe erlaubt sein, meinerseits über das Herrn S. gehörige Arbeitsfeld hinweg zu leuchten: Seine Hauptlehre, daß die Eiweißkörper bei ihrer Umwandlung im Licht bei Anwendung von Katalysatoren eine Sonderstellung einnehmen, ist ja freilich schon von keinem geringeren als C. Neuberg (Berl. Klin. Wochenschr. 1917, Nr. 4) als irrig bewiesen, der HauptsehluB, welchen er aus der photokatalytischen Umwandlung der EiweiBkörper zieht, die Entstehung (oder mindestens starke Förderung) des grauen Stars unter dem Einfluß ultravioletten Lichtes müßte doch aber im 
Verein mit der von Herrn S. immer wieder auts schürfste betonten Zuaahme der ultravioletten Strahlungsintensität mit der Höhe unabwendbar zu der Endfolgerung führen, daB unter den in der Höhe lebenden Menschen der Star viel weiter verbreitet sein muB, als unter den Bewohnern der Ebene. Wie kommt es, daß Herr S. diese Konsequenz nicht zieht? Kann er irgend ein Beweismaterial für diese notwendige Folgerung aufbringen? Daraus, daß er es noch nicht gebracht hat, darf man wohl den Schluß ziehen, daB er keines besitzt und beschaffen kann. Mit großem Nachdruck und in einer auf den Fernerstehenden sehr eindrucksvollen Weise behauptet Sohanz: Die Linse des Auges fluoreseiert - hierbei werden kurzwellige in langwelligere Lichtstrahlen umgewandelt - eine solche Umwandlung der Energie ist undenkbar ohne Veränderung des Mediums, in dem die Umwandlung stattfindet - die Veränderung, welche das Licht an der linse erzengt, summiert sich durch das ganze Leben, denn da sie nerven- und gefäßlos ist, vermag sie nicht Reaktionen auszulösen, welche die durch das Licht verursachten wieder ausgleichen.

Die Fluorescenz ist wesensgleich der Phosphorescenz. Nach Le n a r d s prachtw vollen Untersuchungen deutet die Physik die Lichterscheinung der Phosphorescenz dahin, dab photoelektrisch ausgelöste, aus ihrer Normallage gebrachto Elektronen nach Aufhören der Bestrahlung in die Normallage zurückkehren. Zu dieser selbsttätigen Rüokwandlung bedarf es also gar keiner vitalen Kräfte (keiner Nerven and Gefäße). and eine Summierung der Strahlenwirkungen auf die Linse dureh das ganze Menschenleben hindurch aus der Fluoreseenz abzuleiten, dürfte durchaus verfehlt sein. 